\title{
Applying the Affinto Ontology to Develop a Text-Based Emotional Conversation System
}

\author{
Idoia Cearreta and Nestor Garay \\ Informatika Fakultatea. \\ University of the Basque Country/Euskal Herriko Unibertsitatea, \\ Manuel Lardizabal 1, 20018 Donostia, Spain \\ \{idoia.cearreta, nestor.garay\} @ehu.es
}

\begin{abstract}
With the recent spread of computing systems the need to enhance interactions between users and systems is evident. Conversation systems have a key role to play in achieving this. However, further efforts are needed to enhance conversation systems that use text to interact with users. This paper presents a text conversation system that includes user emotion recognition and generation, with the aim of achieving a more natural communication. The Affinto ontology is used to perform these tasks.
\end{abstract}

Keywords: Conversational System, Affective Computing, Ontology.

\section{Introduction}

With the spread of computing systems in recent years, there is a growing need to enhance interaction with these systems. The main problem when interacting with these systems is the need to take into account the affective values transmitted by users. In addition, a system has to make decisions based on these values and it has to transmit its own emotions. However, this is not an easy task because there are a lot of practical issues to resolve.

In this paper, a text-based emotional conversation system has been developed using the techniques employed in Affective Computing [1]. This involves the development of emotion recognition and generation processes. These processes are developed using an ontology called Affinto [2].

The next section reviews related work, while section 3 describes the components of the conversation system and presents an interaction example. The final section contains the conclusions that can be drawn from this work and the future work.

\section{Related Work}

There are a huge number of conversation systems that provide information and several services to users. In most of these systems the user has to transmit messages via speech and has to be able to receive speech signals transmitted by the systems [3]. Moreover, the majority of conversation systems found in the literature do not take into account the affective values of messages.

With respect to Affective Computing, Baldassarri et al. [4] have developed a conversation interface to enable communication between the user and a real-time 
virtual actor. This communication is performed using natural language and a speech synthesizer that allows the changing of parameters in order to generate affective speech. Unlike Baldassarri et al., in this paper we present a conversation system that recognizes emotions from text. The system also gives text-based affective responses. The abovementioned Affinto ontology is used to associate words with their corresponding affective values. Furthermore, user interactions with the system are gathered in the ontology in order to compile a correctly labelled sentence corpus for conducting further studies in the area of text-only emotion recognition.

There are several methods and techniques for recognizing emotions in texts. In our work we use the technique based on affective dictionaries [5], since the others require a large labelled corpus.

\section{Prototype of an Emotional Conversation System}

\subsection{System Description}

A global architecture has been created based on the Affinto ontology [2]. The main objective of this architecture is the development of multimodal affective resources. A more specific architecture has been created for the system presented in this paper. This specific architecture implements the affective interactions between the user and the system by means of text; i.e. verbal information [2]. This architecture has been useful in creating a prototype conversation system that is able to recognize a user's affective states starting from the verbal information channel.

The Affinto ontology represents the concepts involved in the interactions between people and systems. Moreover, it groups the instances of those concepts to infer the information required by the applications that use this ontology. Affinto also provides the option of representing emotions via different classification theories and the option of indicating whether a stimulus is created internally by a person or is a reaction to an external stimulus. This ontology has been created in the OWL language [6].

A method based on affective dictionaries has been used to develop the text emotion recognizer included in the conversation system. This method is based on searching the affective words of a given sentence on an existing dictionary. These dictionaries store the affective values associated with each word. The ANEW dictionary [7] has been selected for this purpose.

With regard to emotion representation, the system uses a dimensional classification incorporating the SAM (Self-Assessment-Manikin) tool [8]. SAM is a non-verbal pictorial assessment technique that consists of three scales corresponding to three dimensions: Valence, Activation and Dominance.

Depending on the emotion estimated by the recognizer, the system will create an affective response that will also be represented using the abovementioned SAM scales. However, instead of using the images of the three scales to display emotions, the system shows the values of the three dimensions integrated in a single image.

The interpreter used in the presented prototype is RebeccaAIML [9], from the ALICE Project [10]. Several changes have been made in its categories to give responses based not only on the text but also on the user emotions. These categories also incorporate affective values within the responses generated by the interpreter. 


\subsection{Architecture of the Text-Based Emotional Conversation System}

The system able to recognize a user's affective state has several modules. Their functionality is described below:

The system starts the conversation by displaying a greeting message to the user. The user answers with a message typed on the keyboard. Then, the Affective Communication Channel module (i.e. the Verbal Channel) receives the text and sends it to the Information Extraction module. This module performs a syntactic analysis of the message to enable the Interpretation and Response Engine module to emotionally label nouns, adverbs, adjectives and verbs. This task is made possible by using the affective dictionary integrated in the Affinto ontology. To this end, an ontology inference engine is used to extract information about the affective values of words and those that are negation dependent are inverted. Then, the average value of the sentence is calculated in order to obtain an estimate of the user's emotion. This estimate is shown to the user on a form via a SAM image which integrates the three dimensional values. It is possible for the user to correct the values shown, if necessary. In this way, real and corrected data are stored in the ontology.

The ALICE dialogue system has been used to generate answers, changing some of the sentences to incorporate semantics about emotions. Thus, the AIML interpreter will find an appropriate affective response and will make it known via the verbal communication channel on the screen, next to the SAM image that corresponds to the system emotion.

\section{Conclusions and Future Work}

In this paper we have presented a prototype conversation system that uses the verbal communication channel. The method used to recognize affective values is based on the ANEW affective dictionary, by obtaining the average value of the entire sentence after emotionally labelling each word. Thus, a large corpus to classify emotions is not required. Since the message transmission is via text, this type of conversation system may be useful to people who are unable to speak or hear.

Moreover, using the Affinto ontology as a knowledge base that describes interactions through several modalities makes it easier to incorporate other types of affective recognition or synthesis, such as via speech. However, using this type of dictionary for text emotion recognition may have some disadvantages. For example, certain adverbs are used to emphasize or change the superlative grade of following words and thus the global affective emotional value of the sentence could change. Therefore, future work will include a more exhaustive syntactic analysis to detect these types of words.

As well as validating the Affinto ontology, another reason for developing this prototype was to create a small corpus based on the conversations between the users and the system. Since the users emotionally validate the sentences they transmit, it is possible to build a corpus of emotionally labelled sentences. Therefore, the corpus created could be used by a text emotion recognizer that uses other techniques.

At the present time, we are evaluating the affective text recognizer with an experiment with volunteers. In this way, we can analyze the concordances between the volunteer results and the estimates provided by the emotional recognition system. 
Acknowledgments. This research work has received financial support from the Department of Education, Universities and Research of the Basque Government.

\section{References}

1. Picard, R.W.: Affective Computing. MIT Press, Cambridge (1997)

2. Cearreta, I., Garay-Vitoria, N.: Ontology for adapting interactions between humans and systems able to manage affective states. In: 18th UMAP - Workshop on User Models for Motivational Systems. Big Island of Hawaii, USA, pp. 1-12 (2010)

3. Ljunglöf, P., Larsson, S., Mühlenbock, K., Thunberg, G.: TRIK: A talking and drawing robot for children with communication disabilities. In: Proc. Nodalida 2009 - 17th Nordic Conference of Computational Linguistics. Short paper and demonstration (2009)

4. Baldassarri, S., Cerezo, E., Anaya, D.: Interacción emocional con actores virtuales a través de lenguaje natural. In: Macías, J.A., Granollers, A., Latorre, P. (eds.) Interacción'07 - VIII Congreso Internacional de Interacción Persona-Ordenador, pp. 343-352 (2007)

5. Francisco, V., Gervás, P.: Análisis de dependencias para la marcación de cuentos con emociones. Procesamiento de Lenguaje Natural 37, 1135-5948 (2006)

6. OWL (Web Ontology Language). W3C Recommendation, February 10 (2004), http: / / www.w3 . org/2004/OWL/

7. Bradley, M.M., Lang, P.J.: Affective norms for English words (ANEW): Instruction manual and affective ratings. Technical Report C-1. The Center for Research in Psychophysiology, University of Florida (1999)

8. Bradley, M.M., Lang, P.J.: Measuring emotion: The self-assessment manikin and the semantic differential. Journal of Behavior Therapy and Experimental Psychiatry 25, 49-59 (1994)

9. RebeccaAIML. Recuperado el 5 de Abril de (2011), http: / /rebecca-aiml. sourceforge.net/

10. ALICE AI Foundation. Recuperado el 8 de Septiembre de (2010), http: / / www.alicebot.org/about.html 